\title{
A NOTE ON THE STRONG LAW OF LARGE NUMBERS FOR ASSOCIATED SEQUENCES
}

\author{
A. NEZAKATI
}

Received 21 March 2005 and in revised form 27 April 2005

We prove that the sequence $\left\{b_{n}^{-1} \sum_{i=1}^{n}\left(X_{i}-E X_{i}\right)\right\}_{n \geq 1}$ converges a.e. to zero if $\left\{X_{n}, n \geq 1\right\}$ is an associated sequence of random variables with $\sum_{n=1}^{\infty} b_{k_{n}}^{-2} \operatorname{Var}\left(\sum_{i=k_{n-1}+1}^{k_{n}} X_{i}\right)<\infty$ where $\left\{b_{n}, n \geq 1\right\}$ is a positive nondecreasing sequence and $\left\{k_{n}, n \geq 1\right\}$ is a strictly increasing sequence, both tending to infinity as $n$ tends to infinity and $0<a=\inf _{n \geq 1} b_{k_{n}} b_{k_{n+1}}^{-1} \leq$ $\sup _{n \geq 1} b_{k_{n}} b_{k_{n+1}}^{-1}=c<1$.

\section{Introduction}

Let $(\Omega, F, P)$ be a probability space and $\left\{X_{n}, n \geq 1\right\}$ a sequence of random variables defined on $(\Omega, F, P)$. We start with definitions. A finite sequence $\left\{X_{1}, \ldots, X_{n}\right\}$ is said to be associated if for any two componentwise nondecreasing functions $f$ and $g$ on $R^{n}$,

$$
\operatorname{Cov}\left(f\left(X_{1}, \ldots, X_{n}\right), g\left(X_{1}, \ldots, X_{n}\right)\right) \geq 0
$$

assuming of course that the covariance exists. The infinite sequence $\left\{X_{n}, n \geq 1\right\}$ is said to be associated if every finite subfamily is associated. The concept of association was introduced by Esary et al. [1]. There are some results on the strong law of large numbers for associated sequences. Rao [4] developed the Hajek-Renyi inequality for associated sequences and proved the following theorem. Let $\left\{X_{n}, n \geq 1\right\}$ be an associated sequence of random variables with

$$
\sum_{j=1}^{\infty} \frac{\operatorname{Var}\left(X_{j}\right)}{b_{j}^{2}}+\sum_{1 \leq j \neq k}^{\infty} \frac{\operatorname{Cov}\left(X_{j}, X_{k}\right)}{b_{j} b_{k}}<\infty,
$$

where $\left\{b_{n}, n \geq 1\right\}$ is a positive nondecreasing sequence of real numbers. Then $b_{n}^{-1} \sum_{j=1}^{n}$ $\left(X_{j}-E X_{j}\right)$ converges to zero almost everywhere as $n \rightarrow \infty$. In this note we will prove the strong law of large numbers for associated sequences with new conditions. 


\section{Result}

THEOREM 2.1. Let $\left\{X_{n}, n \geq 1\right\}$ be an associated sequence of random variables. If

$$
\sum_{n=1}^{\infty} b_{k_{n}}^{-2} \operatorname{Var}\left(S_{k_{n}}-S_{k_{n-1}}\right)<\infty
$$

where $S_{n}=\sum_{i=1}^{n} X_{i}$ and $\left\{b_{n}, n \geq 1\right\}$ is a positive nondecreasing sequence and $\left\{k_{n}, n \geq 1\right\}$ is a strictly increasing sequence, both tending to infinity as $n$ tends to infinity and

$$
0<a=\inf _{n \geq 1} b_{k_{n}} b_{k_{n+1}}^{-1} \leq \sup _{n \geq 1} b_{k_{n}} b_{k_{n+1}}^{-1}=c<1
$$

Then

$$
\lim _{n \rightarrow \infty} \frac{1}{b_{n}} \sum_{k=1}^{n}\left(X_{k}-E X_{k}\right)=0 \quad \text { a.e. }
$$

Proof. We set $k_{0}=0, b_{0}=0$, and $T_{n}=b_{k_{n}}^{-1} \sum_{j=k_{n-1}+1}^{k_{n}} Y_{j}$, where $Y_{j}=X_{j}-E X_{j}$. For any positive integer $n$, there exists a positive integer $m$ such that $k_{m-1}<n \leq k_{m}$. Note that $m \rightarrow$ $\infty$ as $n \rightarrow \infty$. Without loss of generality, we assume that $n>k_{1}$ and, therefore, $k_{m-1} \geq 1$ and $b_{n} \geq b_{k_{m-1}}>0$. We can show that

$$
\frac{1}{b_{n}} \sum_{j=1}^{n} Y_{j}=\frac{b_{k_{m-1}}}{b_{n}} \sum_{j=1}^{m-1} \frac{b_{k_{j}}}{b_{k_{m-1}}} T_{j}+\frac{1}{b_{n}} \sum_{j=k_{m-1}+1}^{n} Y_{j} .
$$

Since $b_{k_{m-1}} \geq a b_{k_{m}}$, we conclude that

$$
\left|\frac{1}{b_{n}} \sum_{j=1}^{n} Y_{j}\right| \leq\left|\sum_{j=1}^{m-1} \frac{b_{k_{j}}}{b_{k_{m-1}}} T_{j}\right|+\frac{1}{a b_{k_{m}}} \max _{k_{m-1}<l \leq k_{m}}\left|\sum_{j=k_{m-1}+1}^{l} Y_{j}\right| .
$$

In order to prove (2.3) it suffices to demonstrate that each of the two terms in the righthand side of (2.5) converges to zero almost everywhere as $n \rightarrow \infty$. The first term on the right-hand side does so due to the Toeplitz lemma (see Loève [2]) provided that

$$
\lim _{j \rightarrow \infty} T_{j}=0 \quad \text { a.e., } \quad \sup _{m \geq 2} \sum_{j=1}^{m-1} \frac{b_{k_{j}}}{b_{k_{m-1}}}<\infty, \quad \lim _{n \rightarrow \infty} \frac{b_{k_{j}}}{b_{k_{m-1}}}=0 \quad \text { for every } j .
$$

The third condition is satisfied because by the hypothesis the sequence $\left\{b_{n}, n \geq 1\right\}$ monotonically increases without bounds. The second condition holds because

$$
\begin{gathered}
\frac{b_{k_{j}}}{b_{k_{m-1}}}=\prod_{i=j}^{m-2} \frac{b_{k_{i}}}{b_{k_{i+1}}} \leq c^{m-j-1}, \\
\sum_{j=1}^{m-1} \frac{b_{k_{j}}}{b_{k_{m-1}}} \leq \sum_{j=1}^{m-1} c^{m-j-1}=\frac{1-c^{m}}{1-c}<\frac{1}{1-c},
\end{gathered}
$$


since by the hypothesis $b_{k_{j}} \leq c b_{k_{j+1}}, c \in(0,1)$. Thus, the first term in the right-hand side of (2.5) converges to zero almost everywhere as $m \rightarrow \infty$ if the sequence $\left\{T_{n}, n \geq 1\right\}$ also does so. By the hypothesis, Let $\epsilon$ be an arbitrary positive number. With the use of the Markov inequality, we obtain

$$
\epsilon^{2} \sum_{n=2}^{\infty} P\left(\left|T_{n}\right|>\epsilon\right) \leq \sum_{n=2}^{\infty} E\left|T_{n}\right|^{2} \leq \sum_{n=2}^{\infty} b_{k_{n}}^{-2} \operatorname{Var}\left(S_{k_{n}}-S_{k_{n-1}}\right)<\infty .
$$

The finiteness of the last series in the right-hand side is guaranteed by condition (2.1). In view of the Borel-Cantelli lemma, the sequence $\left\{T_{n}, n \geq 1\right\}$ converges to zero a.e. Let us turn to the second term in the right-hand side of (2.5). Applying Chebyschev's inequality, we get that, for any $\epsilon>0$,

$$
\epsilon^{2} P\left(\frac{1}{b_{k_{m}}} \max _{k_{m-1}<l \leq k_{m}}\left|\sum_{j=k_{m-1}+1}^{l} Y_{j}\right|>\epsilon\right) \leq \frac{1}{b_{k_{m}}^{2}} E\left(\max _{k_{m-1}<l \leq k_{m}}\left|\sum_{j=k_{m-1}+1}^{l} Y_{j}\right|^{2}\right) .
$$

We now apply the Kolmogorov-type inequality, valid for partial sums of associated random variables $\left\{Y_{j}, k_{m-1}+1 \leq j \leq k_{m}\right\}$ with mean zero (cf. Newman and Wright [3, Theorem 2]). Hence, from (2.1), we have

$$
\begin{aligned}
\epsilon^{2} \sum_{m=2}^{\infty} P\left(\frac{1}{b_{k_{m}}} \max _{k_{m-1}<l \leq k_{m}}\left|\sum_{j=k_{m-1}+1}^{l} Y_{j}\right|>\epsilon\right) & \leq \sum_{m=2}^{\infty} \frac{1}{b_{k_{m}}^{2}} E\left[\sum_{j=k_{m-1}+1}^{k_{m}} Y_{j}\right]^{2} \\
& \leq \sum_{m=2}^{\infty} \frac{\operatorname{Var}\left(\sum_{j=k_{m-1}+1}^{k_{m}} Y_{j}\right)}{b_{k_{m}}^{2}} \\
& \leq \sum_{m=2}^{\infty} \frac{\operatorname{Var}\left(S_{k_{m}}-S_{k_{m-1}}\right)}{b_{k_{m}}^{2}}<\infty .
\end{aligned}
$$

By virtue of the Borel-Cantelli lemma, the sequence

$$
\left\{\frac{1}{b_{k_{m}}} \max _{k_{m-1}<l \leq k_{m}}\left|\sum_{j=k_{m-1}+1}^{l} Y_{j}\right|\right\}_{m \geq 1}
$$

converges to zero almost everywhere. Thus, the theorem is proved.

THeOREM 2.2. Let $\left\{X_{n}, n \geq 1\right\}$ be an associated sequence of random variables with

$$
\operatorname{Var}\left(X_{j}\right)+\sum_{1 \leq k \neq j}^{\infty} \operatorname{Cov}\left(X_{j}, X_{k}\right)=O(1)
$$

for all $j \geq 1$. Then

$$
\frac{\sum_{j=1}^{n}\left(X_{j}-E X_{j}\right)}{(n \log n)^{1 / 2} \log \log n} \longrightarrow 0 \quad \text { a.e. as } n \longrightarrow \infty \text {. }
$$


Proof. Under condition (2.12), there exists the constant of $B$ such that

$$
\operatorname{Var}\left(S_{k_{n}}-S_{k_{n-1}}\right) \leq B\left(k_{n}-k_{n-1}\right) \leq B k_{n}
$$

The sequence $b_{n}=(n \log n)^{1 / 2} \log \log n$ and $k_{n}=2^{n+1}, n=1,2, \ldots$, satisfy the hypotheses of Theorem 2.1, which proves Theorem 2.2.

Example 2.3. Let $\left\{X_{n}, n \geq 1\right\}$ be an associated sequence with $\operatorname{Var}\left(X_{i}\right)=1$ and $\operatorname{Cov}\left(X_{i}, X_{j}\right)$ $=\rho^{|i-j|}, 0<\rho<1$ for every $i$ and $j$. Then

$$
\operatorname{Var}\left(X_{i}\right)+\sum_{1 \leq j \neq i}^{\infty} \operatorname{Cov}\left(X_{i}, X_{j}\right) \leq 1+2 \sum_{k=1}^{\infty} \rho^{k}<\infty .
$$

Therefore, we can apply Theorem 2.2.

\section{Acknowledgment}

This note was supported by the Shahrood University of Technology in 2004.

\section{References}

[1] J. D. Esary, F. Proschan, and D. W. Walkup, Association of random variables, with applications, Ann. Math. Statist. 38 (1967), 1466-1474.

[2] M. Loève, Probability Theory. Foundations. Random Sequences, D. Van Nostrand, New York, 1955.

[3] C. M. Newman and A. L. Wright, An invariance principle for certain dependent sequences, Ann. Probab. 9 (1981), no. 4, 671-675.

[4] B. L. S. Prakasa Rao, Hajek-Renyi-type inequality for associated sequences, Statist. Probab. Lett. 57 (2002), no. 2, 139-143.

A. Nezakati: Faculty of Mathematics, Shahrood University of Technology, Shahrood, P.O. Box 36155-316, Iran

E-mail address: nezakati@shahrood.ac.ir 


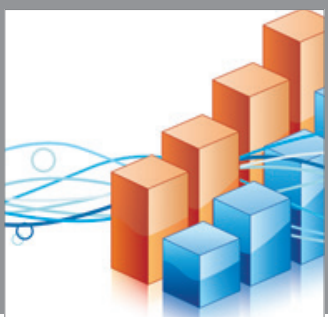

Advances in

Operations Research

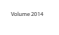

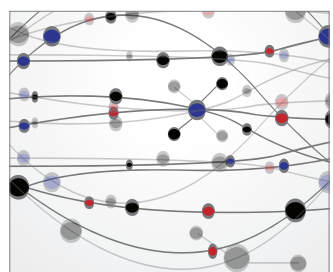

\section{The Scientific} World Journal
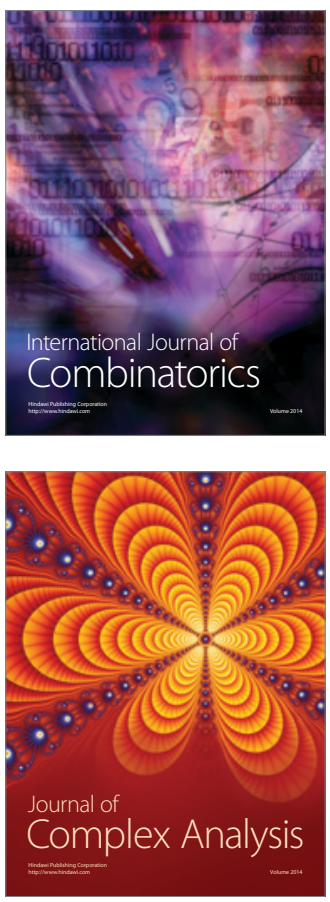

International Journal of

Mathematics and

Mathematical

Sciences
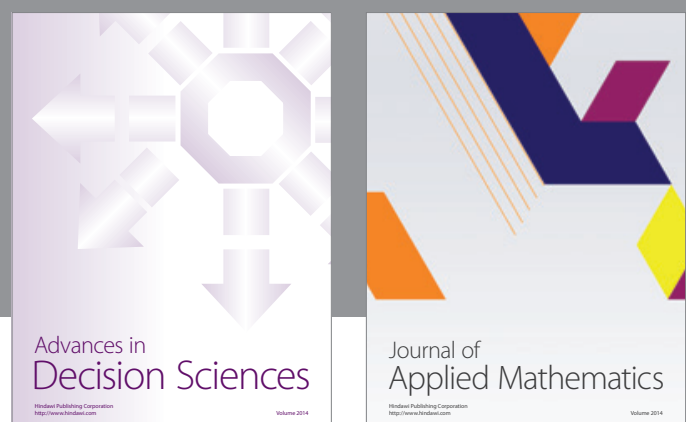

Journal of

Applied Mathematics
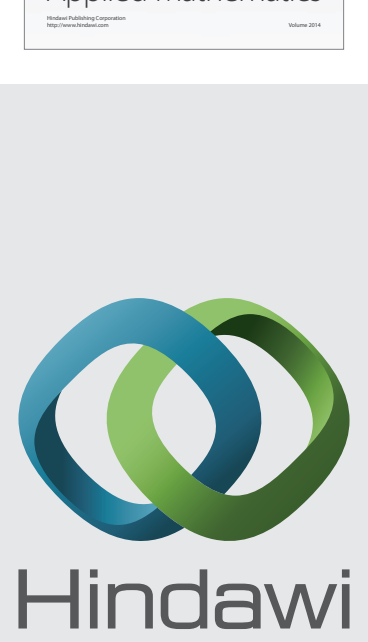

Submit your manuscripts at http://www.hindawi.com
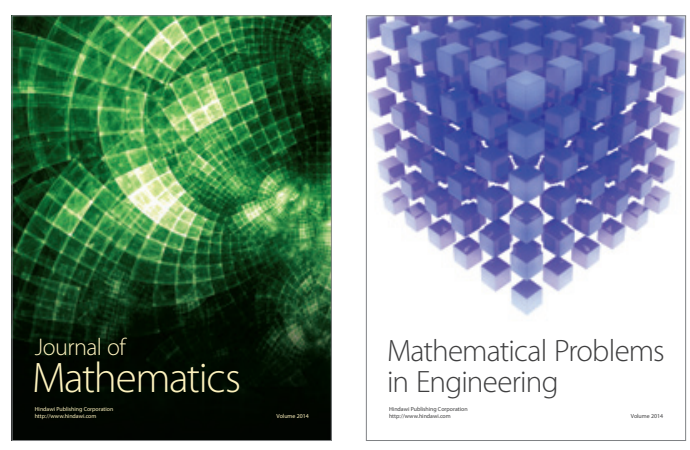

Mathematical Problems in Engineering
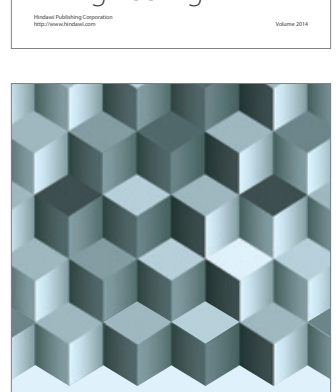

Journal of

Function Spaces
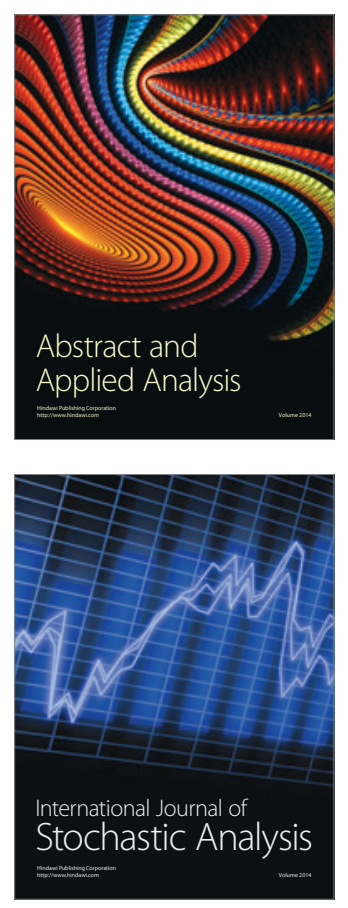

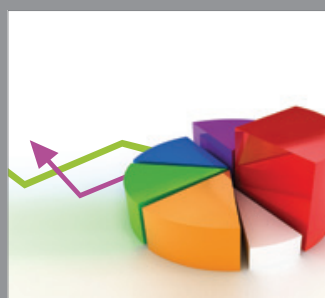

ournal of

Probability and Statistics

Promensencen
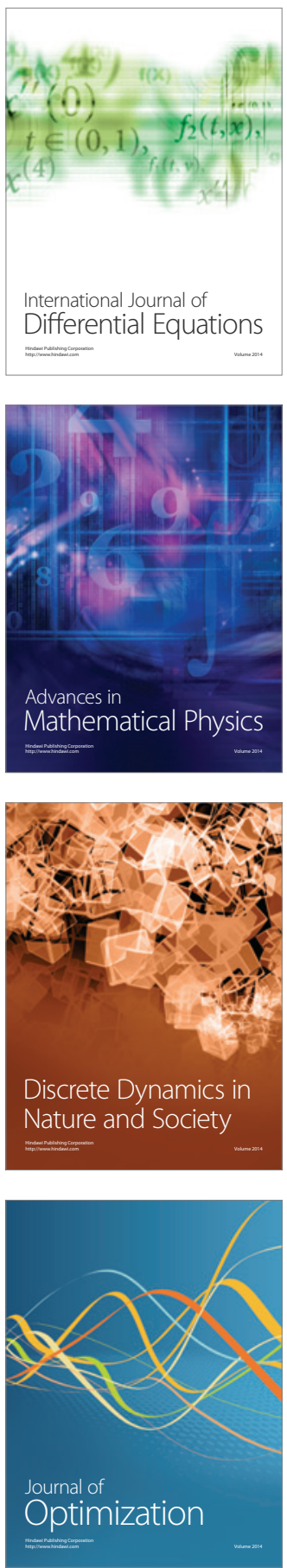